\title{
"Unforgettable" - a pictorial essay on anatomy and pathology of the hippocampus
}

\author{
Sven Dekeyzer ${ }^{1,2,3}$ (D) Isabelle De Kock ${ }^{2}$ - Omid Nikoubashman ${ }^{1}$. \\ Stephanie Vanden Bossche ${ }^{2} \cdot$ Ruth Van Eetvelde ${ }^{2,3} \cdot$ Jeroen De Groote $^{2} \cdot$ Marjan Acou $^{2}$ • \\ Martin Wiesmann ${ }^{1} \cdot$ Karel Deblaere $^{2} \cdot$ Eric Achten $^{2}$
}

Received: 19 September 2016/Revised: 18 December 2016 / Accepted: 20 December 2016 / Published online: 20 January 2017

(C) The Author(s) 2017. This article is published with open access at Springerlink.com

\begin{abstract}
The hippocampus is a small but complex anatomical structure that plays an important role in spatial and episodic memory. The hippocampus can be affected by a wide range of congenital variants and degenerative, inflammatory, vascular, tumoral and toxic-metabolic pathologies. Magnetic resonance imaging is the preferred imaging technique for evaluating the hippocampus. The main indications requiring tailored imaging sequences of the hippocampus are medically refractory epilepsy and dementia. The purpose of this pictorial review is threefold: (1) to review the normal anatomy of the hippocampus on MRI; (2) to discuss the optimal imaging strategy for the evaluation of the hippocampus; and (3) to present a pictorial overview of the most common anatomic variants and pathologic conditions affecting the hippocampus.
\end{abstract}

\section{Teaching points}

- Knowledge of normal hippocampal anatomy helps recognize anatomic variants and hippocampal pathology.

- Refractory epilepsy and dementia are the main indications requiring dedicated hippocampal imaging.

- Pathologic conditions centered in and around the hippocampus often have similar imaging features.

Sven Dekeyzer

sven.dekeyzer@gmail.com

1 Department of Diagnostic and Interventional Neuroradiology, University Hospital, RWTH Aachen University, Pauwelsstr. 30, 52074 Aachen, Germany

2 Department of Radiology, University Hospital (UZ) Ghent, De Pintelaan 185, 9000 Ghent, Belgium

3 Department of Medical Imaging, Onze-Lieve-Vrouw Hospital (OLV) Aalst, Moorselbaan 164, 9300 Aalst, Belgium
- Clinical information is often necessary to come to a correct diagnosis or an apt differential.

Keywords Hippocampus $\cdot$ Epilepsy $\cdot$ Dementia $\cdot$ Herpes simplex encephalitis $\cdot$ MRI

$\begin{array}{ll}\text { Abbreviations } \\ \text { AD } & \text { Alzheimer's dementia } \\ \text { DNET } & \text { Dysembryoblastic neuroepithelial tumour } \\ \text { IHI } & \text { Incomplete hippocampal inversion } \\ \text { HSE } & \text { Herpes simplex encephalitis } \\ \text { LE } & \text { Limbic encephalitis } \\ \text { MTA } & \text { Mesial temporal atrophy } \\ \text { MTS } & \text { Mesial temporal sclerosis }\end{array}$

Anatomy, embryology, arterial supply and function

\section{Normal hippocampal anatomy}

The hippocampus is a bilaminar gray matter structure located medially in the temporal lobe that protrudes over the temporal horn of the lateral ventricle and occupies the medial region of its floor (Figs. 1 and 2). The hippocampus consists of two interlocking gray matter folds, the cornu ammonis (or hippocampus proper) and the dentate gyrus. In the axial plane, the hippocampus resembles a seahorse (hence, its name) and it arches around the mesencephalon (hence, the term "mesiotemporal"). In the axial and sagittal plane, it can be divided into three parts: (1) the head or anterior segment; (2) the body or intermediate segment; and (3) the tail or posterior segment. White matter fibres from the hippocampus accumulate on its superior surface to form the alveus. White matter fibres from the alveus 

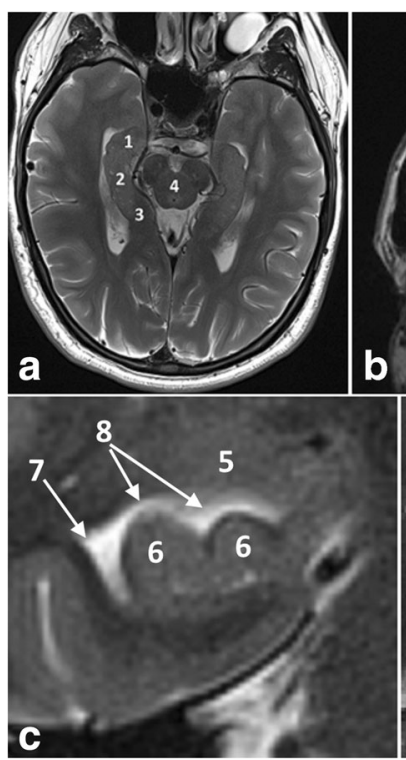
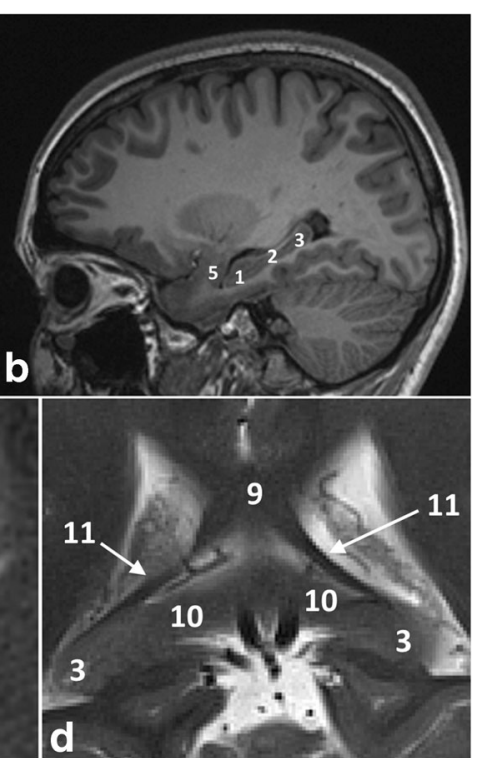

Fig. 1 Anatomy of the hippocampal formation on 3-T axial T2 (a) and sagittal 3D-MPRAGE images (b). Zoomed-in 3-T coronal T2-weighted images at the level of the hippocampal head (c) and the hippocampal tail (d). The hippocampal body is shown in detail in Fig. 2. 1= hippocampal head, 2 = hippocampal body, $3=$ hippocampal tail, $4=$ mesencephalon, $5=$ amygdala, $6=$ hippocampal digitations, $7=$ temporal horn of the lateral ventricle, $8=$ uncal recess of the lateral ventricle, $9=$ splenium of the corpus callosum, $10=$ subsplenial gyri, $11=$ crura of the fornices. To easily recognize the different portions of the hippocampus, we can use the mesencephalon (4). The head (1) is located in front of the mesencephalon, the body (2) can be found at the level of the mesencephalon and the tail (3) is posterior to the mesencephalon. The hippocampal head is the only portion of the hippocampus not covered by the choroid plexus (7). The hippocampal head is separated from the amygdala (5) by the uncal recess of the lateral ventricle (7) and is characterized by small digitations separated by small sulci, the digitationes hippocampi (6). At the level of the hippocampal tail, the fimbriae continue posteriorly as the crux of the fornix (11) that slants upwards towards the splenium of the corpus callosum (9) and the hippocampal tail continues as the subsplenial gyri (10).

then gather medially into thickened bundles as the fimbria, which are continuous posteriorly with the fornix [1].

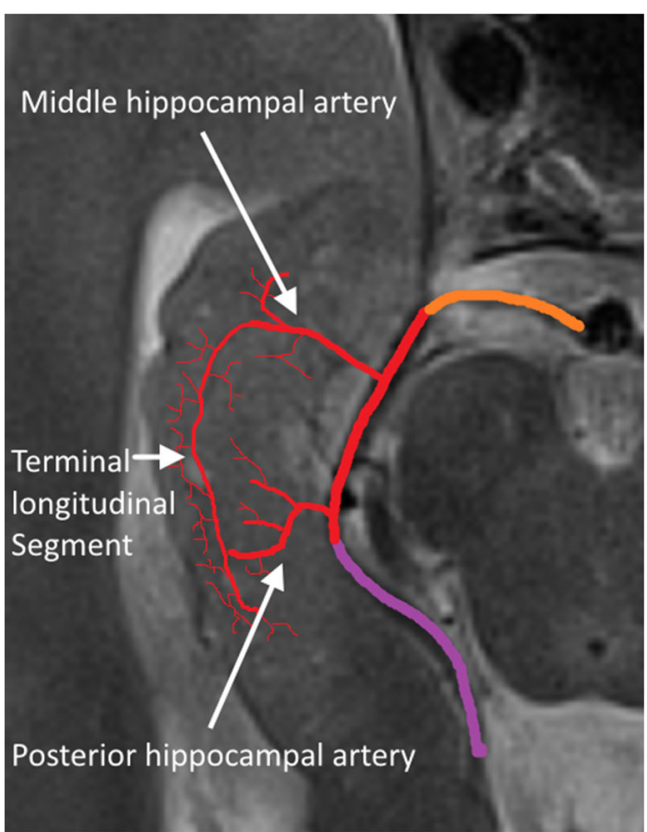

Fig. 3 Arterial supply of the hippocampal body and tail. Orange $=\mathrm{P} 1$, red $=\mathrm{P} 2$ and purple $=\mathrm{P} 3$ segment of the posterior cerebral artery. The anterior hippocampal artery is hidden in the uncal sulcus and is shown in Fig. 4

Based on its cellular composition, the cornu ammonis is divided into four parts, the so-called Sommer's sectors CA1 to CA4. The cornu ammonis continues inferomedially in the parahippocampal gyrus, a gray matter structure that forms the transition area between the basal and mesial areas of the temporal lobe. The subiculum is the medial and superior edge of the parahippocampal gyrus and its site of union with the cornu ammonis[1].The hippocampus is surrounded by several fissures which are collectively referred to as the perihippocampal fissures. The transverse fissure of Bichat is the lateral extension of the ambient cistern which separates the thalamus superiorly from the parahippocampal gyrus inferiorly. The superolateral extension of the transverse fissure is the choroidal fissure. The

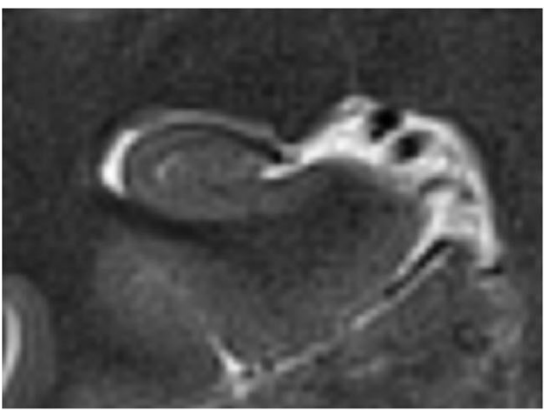

Fig. 2 Anatomy of the hippocampal formation at the level of the hippocampal body on 3-T coronal T2. The hippocampal formation consists of the cornu ammonis or hippocampus proper, which can histologically be divided in the four Sommer sectors CA1-CA4, and the dentate gyrus (DG). A small hippocampal cyst (Hs) reflects the location of the largely obliterated hippocampal sulcus. A=alveus,

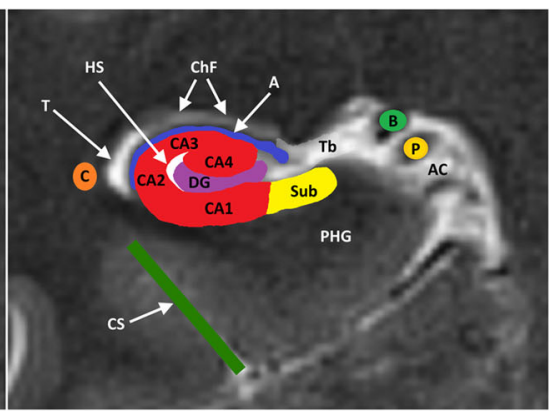

$\mathrm{Ac}=$ Ambient cistern, $\mathrm{B}=$ basal vein of Rosenthal, $\mathrm{C}=$ tail of caudate nucleus, $\mathrm{ChF}=$ choroid fissure, $\mathrm{CS}=$ collateral suclus, $\mathrm{DG}=$ dentate gyrus, $\mathrm{P}=$ posterior cerebral artery, $\mathrm{PHG}=$ parahippocampal gyrus, $\mathrm{Sub}=$ subiculum, $\mathrm{T}=$ temporal horn of the lateral ventricle, $\mathrm{Tb}=$ transverse fissure of Bichat 


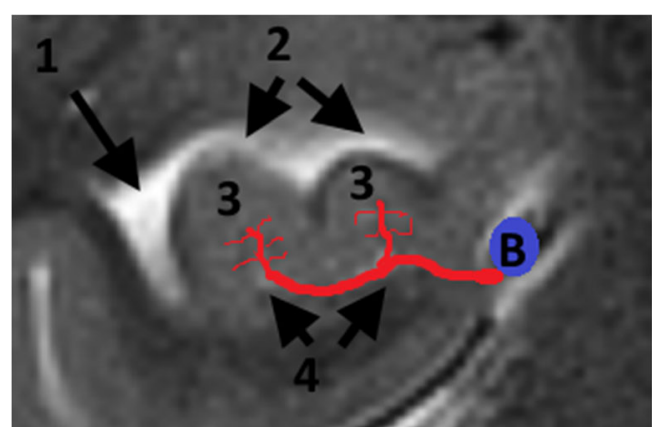

Fig. 4 Arterial supply of the hippocampal head. $B=$ basal Rosenthal vein, $1=$ temporal horn of the lateral ventricle, $2=$ uncal recess of the lateral ventricle, $3=$ hippocampal digitations, $4=$ uncal sulcus. Both the anterior hippocampal artery, originating from the trunk or branches of the posterior cerebral artery, and the uncal branch of the anterior choroidal artery, dive into the uncal sulcus at the level of the hippocampal head and form anastomoses in the sulci between the hippocampal digitations. Here, only one of both arteries in the uncal sulcus is drawn

inferolateral extension of the transverse fissure is the hippocampal fissure, which extends between the dentate gyrus and the subiculum and is often obliterated and not visible on MRI [1].

\section{Arterial supply of the hippocampus}

Usually, three arteries (or groups of arteries) arising from the main or branches of the posterior cerebral artery vascularize the hippocampus: the anterior, middle and posterior hippocampal arteries. The anterior hippocampal artery supplies the hippocampal head, whereas the middle and posterior hippocampal arteries vascularize the hippocampal body and tail. The middle and posterior hippocampal artery are richly interconnected with another through the so-called longitudinal terminal segments that run parallel to the course of the hippocampal body (Fig. 3). The uncal branch of the anterior choroidal artery is usually anastomosed with the anterior hippocampal artery in the uncal sulcus (Fig. 4) [1].

\section{Normal hippocampal development}

The bulk of the cerebral cortex consists of the neocortex, a phylogenetically advanced structure consisting of six cell layers. The mesial temporal structures are formed of more primitive allocortex with three, four or five cell layers and begin their development in early fetal life as a flat cortical
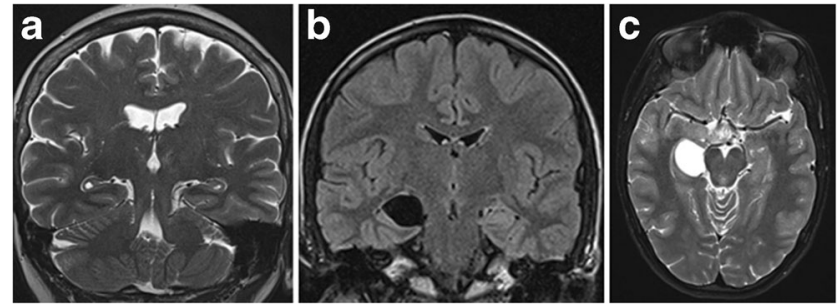

Fig. 6 Bilateral sulcal remnant cysts (a) and right-sided choroid fissure cyst $(\mathbf{b}, \mathbf{c})$. Coronal T2 shows small bilateral cysts at the apex of the hippocampal fold between the dentate gyrus and Ammon's horn (a). Coronal FLAIR (b) and axial T2-weighted (c) images show a spaceoccupying cystic lesion, iso-intense to cerebrospinal fluid, at the level of the right choroid fissure

plate along the medial wall and floor of the temporal horn (Fig. 5a). Gradual infolding of the various components occurs as a result of expansion of the neocortex and unequal growth of the various hippocampal components (Fig. 5b, c). The infolding occurs around the hippocampal sulcus, a sulcus that develops between the dentate gyrus and cornu ammonis and later shifts to a location between the dentate gyrus and subiculum (Fig. 5d). Eventually, the hippocampal sulcus becomes obliterated, but at times, a residual cavity, a so-called sulcal remnant cyst, may be seen (Fig. 6a).

\section{Function}

The hippocampus plays an important role in spatial and episodic memory. A possible role of the medial temporal lobe in memory formation was first suggested over a century ago by von Bechterew after presenting the neuropathological findings from the brain of a 60 -year-old man who had memory problems during the last 20 years of his life and whose brain showed bilateral softening of the gyrus uncinatus and the hippocampal formation [2]. The role of the hippocampus in episodic memory became firmly established in 1957, when Scoville described a case of severe antegrade amnesia following bilateral medial temporal lobe resection in a patient with intractable seizures [3].

\section{Imaging protocol}

The design of the imaging protocol largely depends on the clinical question. Indications that require tailored sequences
Fig. 5 Embryologic development of the hippocampus (image taken from Atlas klinische Neuroradiologie des Gehirns, Springer Berlin Heidelberg, 2011, p. 23, by Lin, Wiesmann and Brückmann. With permission of Springer (C)

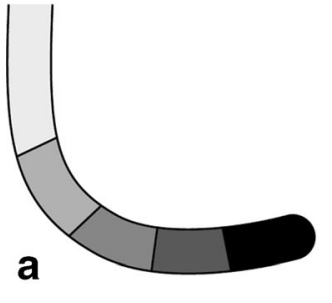

- Parahippocampal gyrus
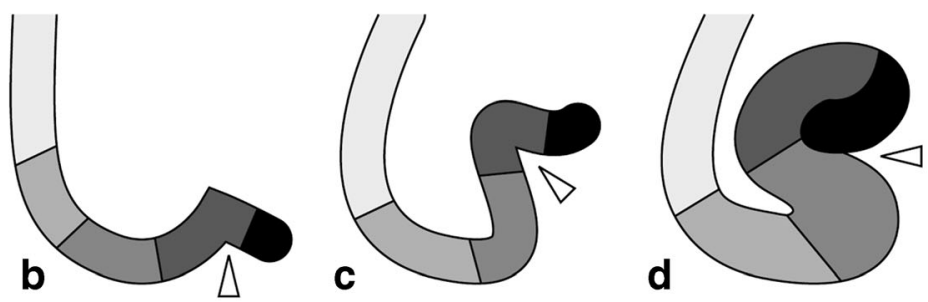

Subiculum $\quad$ Hippocampus proper

$\triangle$ Hippocampal sulcus 


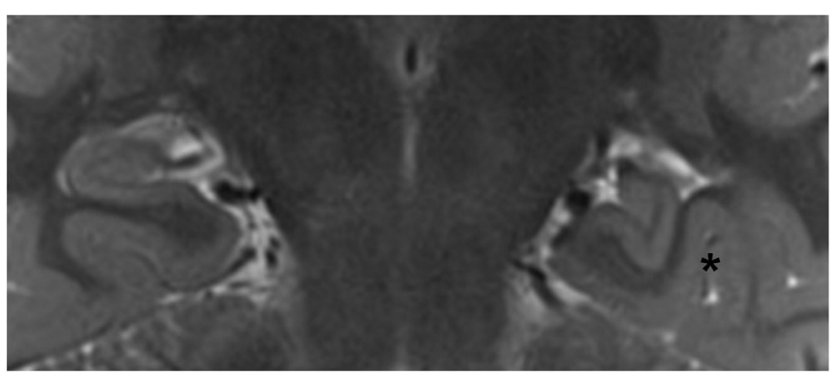

Fig. 7 Isolated IHI. Coronal 3-T T2-weighted image in a 23-year-old patient with epilepsy shows a normal hippocampus on the right compared to an incompletely inverted hippocampus with an abnormal rounded or pyramidal shape on the left. The left collateral sulcus (asterisk) has a more vertical orientation and is found lateral of the hippocampal body

for optimal visualization of the hippocampus are medically refractory epilepsy and dementia $[4,5]$.

In patients with medically refractory epilepsy, MRI at $3 \mathrm{~T}$, if available, is preferred, and for the hippocampus, a 1-mm isotropic 3D series with T1W and FLAIR contrast with a good signal-to-noise ratio must be acquired with reconstructions along and perpendicular to the plane of the hippocampi $[6$, 7]. A tilted coronal $\mathrm{T} 2$-weighted (T2W) series should be acquired as well with high resolution $(0.5 \mathrm{~mm}$ or less in-plane) to evaluate the internal hippocampal structure. If acquisition time is limited, at least coronal IRT1W $(1.5 \mathrm{~T})$ or T2W (3 T) images perpendicular to the hippocampal plane should be acquired as extra to a standard screening protocol [8]. Contrast administration is only necessary if findings on the nonenhanced series need further investigation [4].

For an optimal evaluation of the hippocampi in patients with dementia, a 3D T1W sequence with coronal reconstructions perpendicular to the plane of the hippocampi is
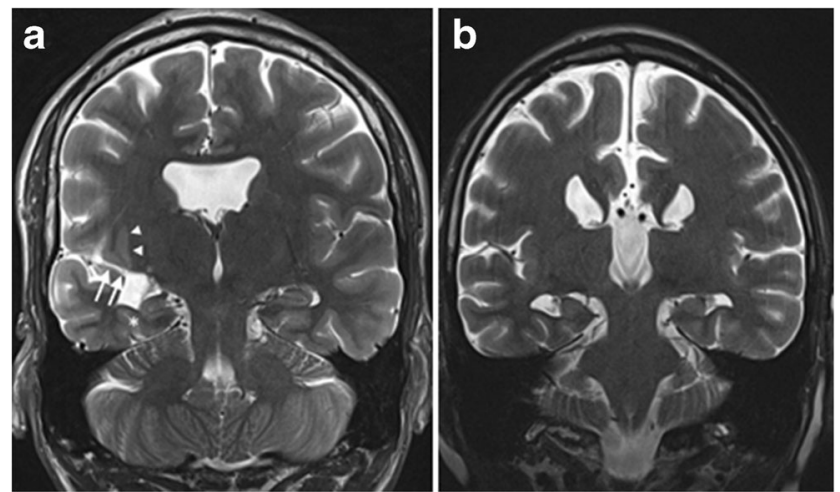

Fig. 8 IHI associated with other developmental anomalies. Coronal T2weighted image in a 22 -year-old male patient with mental retardation and epilepsy (a) shows incomplete inversion of the right hippocampus (white asterisk at the collateral sulcus) along with a right perisylvian open lip schizencephaly (white arrows), right perisylvian subependymal heterotopia (white arrowheads) and agenesis of the septum pellucidum. Coronal T2-weighted images in a 25 -year-old patient with mental retardation and epilepsy (b) shows agenesis of the corpus callosum and an abnormal globular shape of both hippocampi, corresponding to a bilateral incomplete hippocampal inversion

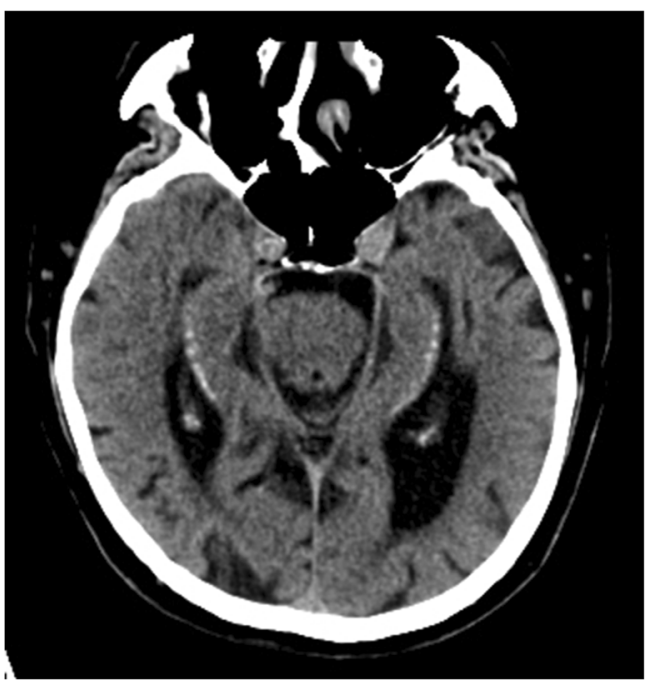

Fig. 9 Hippocampal calcifications. Non-enhanced axial CT images angulated parallel to the hippocampal body show bilateral symmetrical calcifications lateral in the hippocampal bodies as a coincidental finding in a 69-year-old female patient with dysarthria

recommended. If 3D techniques are unavailable, coronaloblique 2D T1W images can serve as an alternative [5].

\section{Imaging features of anatomic variants and pathologic entities}

\section{Congenital anomalies}

\section{Sulcal remnant cysts and choroidal fissure cysts}

Sulcal remnant cysts and choroidal fissure cysts are benign cerebral cysts that can occasionally be found at the level of the vestigial hippocampal sulcus and the choroid fissure, respectively (Fig. 6). Sulcal remnant cysts are residual cysts resulting from lack of obliteration of the hippocampal sulcus
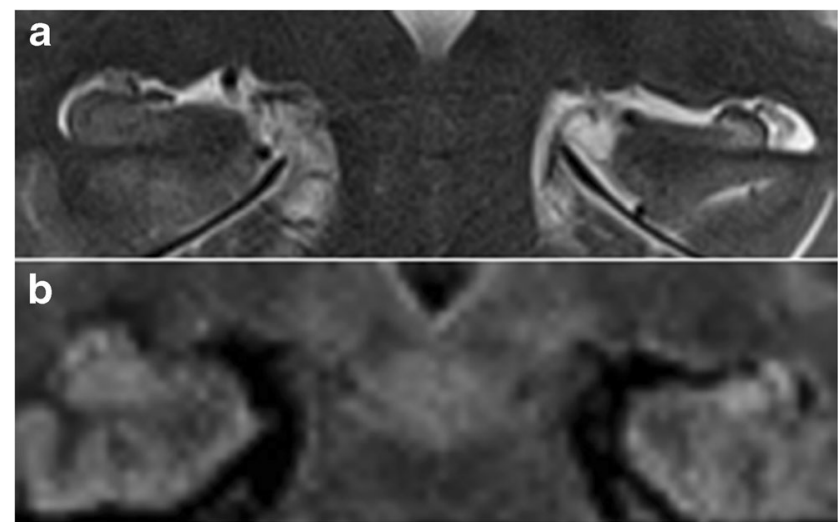

Fig. 10 Left-sided mesial temporal sclerosis. $3 \mathrm{~T}$ coronal T2-weighted (a) and FLAIR images (b) in a 43-year-old patient with medically refractory epilepsy show volume loss, increased signal intensity and blurring of the internal structure of the left hippocampus, compatible with mesial temporal sclerosis 

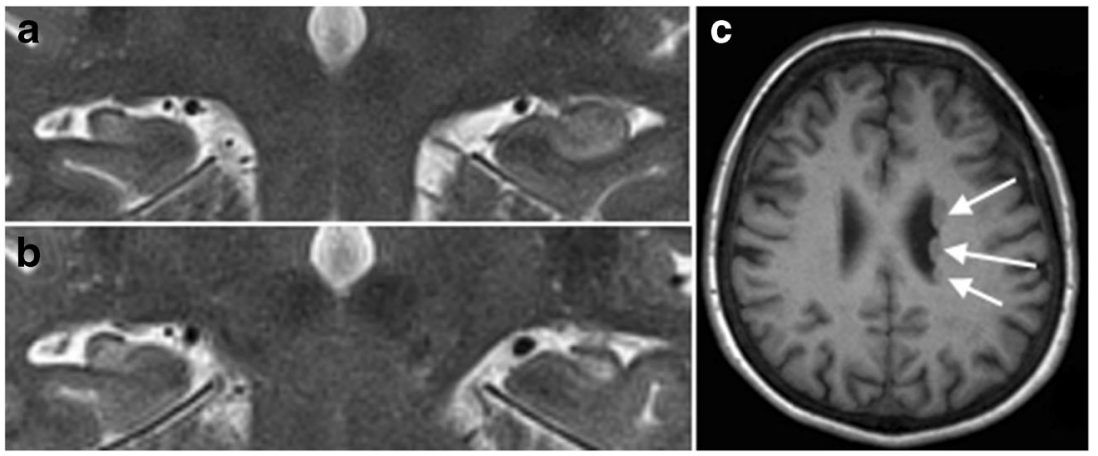

Fig. 11 Bilateral hippocampal sclerosis and dual pathology. 3-T coronal T2-weighted image in a 35-year-old patient with medically refractory epilepsy shows volume loss and increased signal intensity of the right hippocampus, compatible with mesial temporal sclerosis (a). The left side the hippocampal volume looks normal, but $\mathrm{T} 2$ signal intensity is slightly increased in the CA1 and CA2 sectors and there is blurring of the internal structure (a). On follow-up MRI performed 3 years later, there is clear bilateral hippocampal sclerosis with atrophy and increased T2 signal intensity of both hippocampi (b). Axially reconstructed 3D MPRAGE images (c) show multiple periventricular heterotopias (white arrows) along the lateral wall of the left lateral ventricle

described in patients with epilepsy and/or severe midline malformations and other developmental malformations, but is also seen in up to $19 \%$ of nonepileptic patients without other intracranial abnormalities [10,11]. In patients with epilepsy, $\mathrm{IHI}$ is not considered an epileptogenic lesion per se, but rather a sign of a more widespread disorder of cerebral development that may affect other parts of the brain leading to epilepsy.

\section{Degenerative diseases}

\section{Hippocampal calcifications}

Hippocampal calcifications are a frequent incidental finding on CT (Fig. 9). Their prevalence increases with age and they are described in up to $21.7 \%$ of the population over 50 years of age. Hippocampal calcifications are not associated with neurodegenerative diseases. Their pathologic significance is unclear, but they most likely reflect the latter stages of vascular fibrosis [12].

\section{Mesial temporal sclerosis}

The most common cause of medically intractable partial complex epilepsy in adults is mesial temporal sclerosis (MTS). MTS is characterized pathologically by hippocampal gliosis and neuronal loss. The pathophysiology of MTS is not
Table 1 Visual rating scale of mesial temporal atrophy. $\mathrm{N}=$ normal; $\uparrow=$ increase; $\downarrow=$ decrease

\begin{tabular}{llll}
\hline MTA Score & Width of choroid fissure & Width of temporal horn & Height of hippocampal formation \\
\hline 0 & $\mathrm{~N}$ & $\mathrm{~N}$ & $\mathrm{~N}$ \\
1 & $\uparrow$ & $\mathrm{N}$ & $\mathrm{N}$ \\
2 & $\uparrow \uparrow$ & $\uparrow$ & $\downarrow$ \\
3 & $\uparrow \uparrow \uparrow$ & $\uparrow \uparrow$ & $\downarrow \downarrow$ \\
4 & $\uparrow \uparrow \uparrow$ & $\uparrow \uparrow \uparrow$ & $\downarrow \downarrow \downarrow$ \\
\hline
\end{tabular}


Fig. 12 The Scheltens mesial temporal atrophy scale in coronal 3D-MPRAGE images (images from the Radiology Assistant website with permission http://www.radiologyassistant.nl)

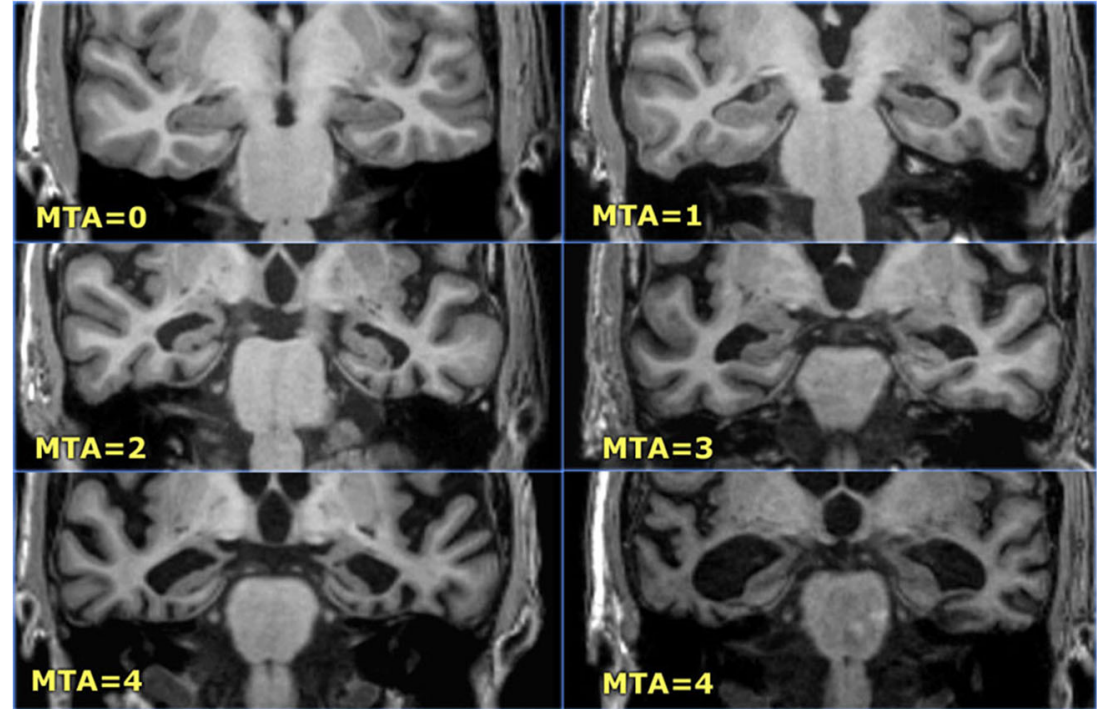

completely understood. One theory is that early or prolonged febrile seizures damage the hippocampus in genetically susceptible patients. However, the difference between cause and effect is not clear, as it is also conceivable that a child may have prolonged febrile seizures due to MTS secondary to a prenatal/perinatal insult or genetic predisposition [13, 14].

Neuronal cell loss and gliosis result in the MRI findings of hippocampal atrophy with increased T2/FLAIR signal intensity (Fig. 10). Other secondary signs can be present such as: (1) loss of the internal architecture of the hippocampus; (2) loss of hippocampal head digitations; and (3) dilatation of the ipsilateral temporal horn. Because of physiologic interconnections between the hippocampus and other parts of the brain, secondary signs can also involve other structures such as: (1) increased signal intensity and/or atrophy of the ipsilateral amygdala; (2) atrophy of the ipsilateral mammillary body; (3) atrophy of the ipsilateral fornix; (4) atrophy of the contralateral cerebellar hemisphere; and (5) atrophy of the ipsilateral entorhinal area [15-17].

Identification of MTS is important as surgery is often the only treatment option with a good outcome. Regarding the radiological pre-surgical workout, it is important to note that MTS can be bilateral in up to 3-10\% of cases although symptomatology may be unilateral [18], and that dual pathology, the existence of a second epileptogenic lesion, can be present in up to $15 \%$ of cases (Fig. 11) [19].

\section{Alzheimer's disease and other dementias}

Alzheimer's disease Alzheimer's disease (AD) is a progressive neurodegenerative disorder that is characterised pathologically by an accumulation of senile plaques and neuritic tangles. It typically begins in the transenthorinal regions and spreads to the hippocampal cortex and mesial temporal lobes before progressing to the temporal lobes, basal forebrain and the rest of the brain.

Over the past decades, the role of imaging in dementia has shifted from merely excluding possible treatable causes of dementia (e.g. tumour or subdural hematoma) to the identification of certain positive disease markers. In this regard, the mesial temporal atrophy (MTA) score, a radiologic measure for the degree of hippocampal atrophy, has proven to be predictive of progression from mild cognitive impairment to $\mathrm{AD}$ [20] and can identify patients with AD with an accuracy and sensitivity of approximately $85 \%$ compared to healthy controls [21]. The MTA score is based on four points and takes into account the width of the temporal horn of the lateral ventricle, the width of the choroid fissure and the hippocampal volume (Table 1, Fig. 12) [20]. For subjects younger than 75 years, an MTA score of 2 or more is considered abnormal, while for subjects older than 75 years, an MTA score of 3 or more is considered abnormal [21]. Its usefulness for the

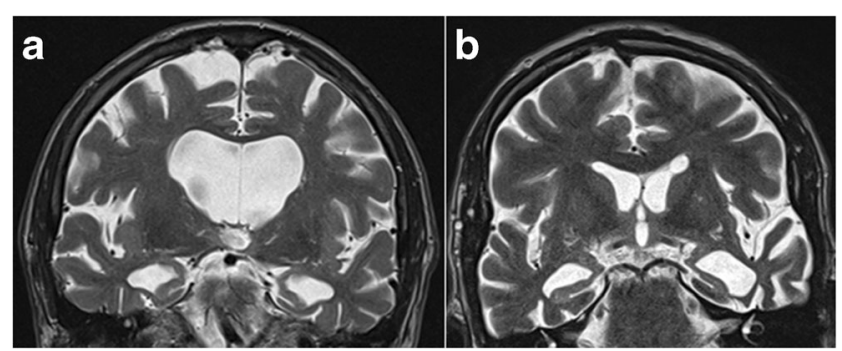

Fig. 13 Alzheimer's disease (a) and frontotemporal dementia (b). 3-T Coronal T2-weighted images in an 84-year-old patient with clinically advanced sporadic Alzheimer dementia show pronounced mesial temporal atrophy corresponding to an MTA score of 4 (a). In comparison, coronal T2-weighted images in a 58-year-old patient with a semantic variant of frontotemporal dementia also shows pronounced bilateral mesiotemporal atrophy (MTA score 4), along with asymmetric pronounced cortical atrophy of the left temporal neocortex (b) 


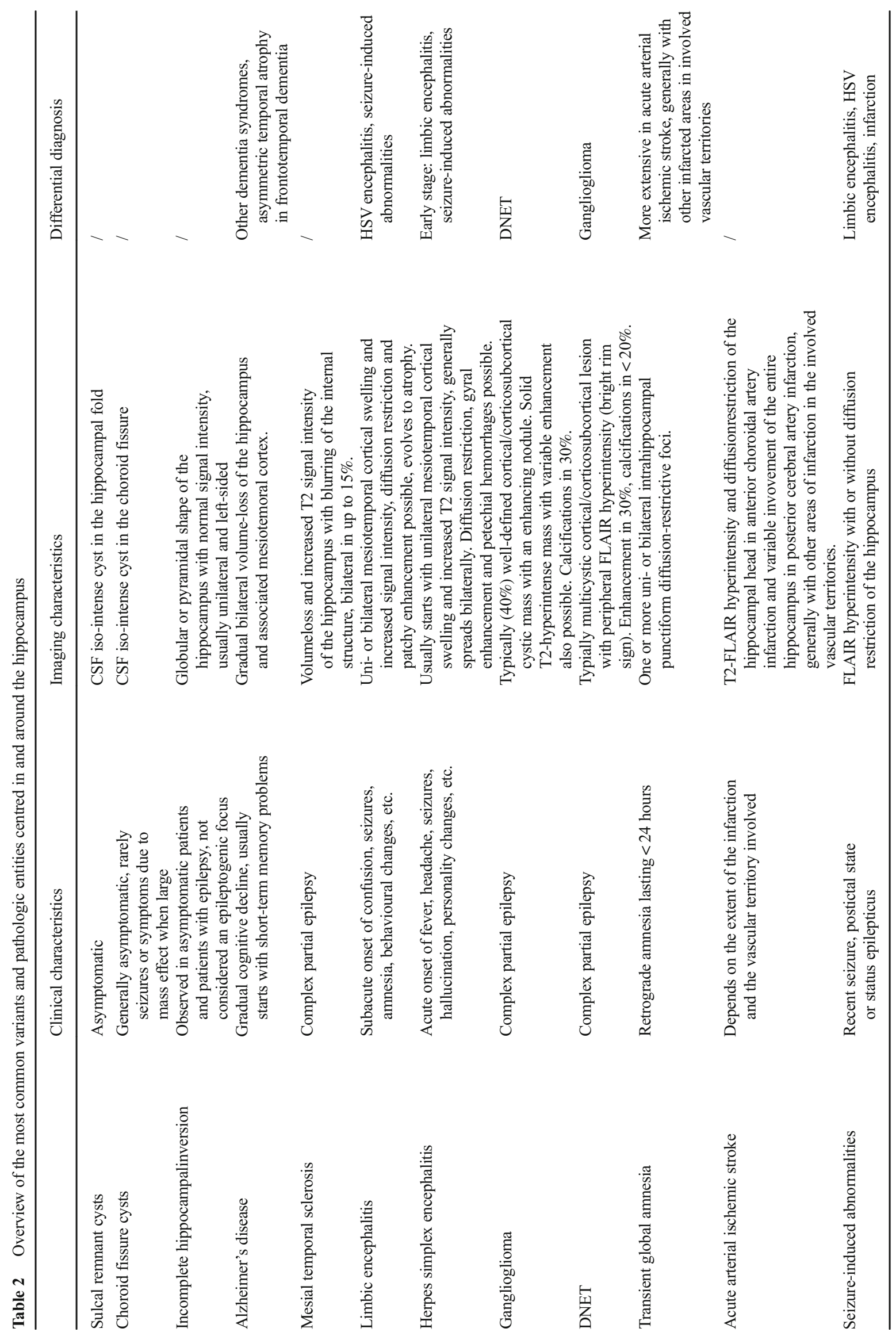




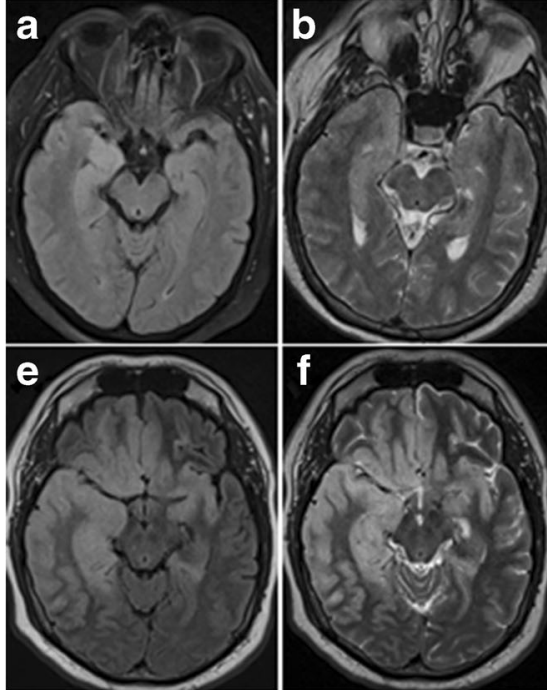

Fig. 14 Early stage (a-d) versus more advanced $(\mathbf{e}-\mathbf{h})$ herpes encephalitis. 44-year-old male patient with fever, headache and acute epileptic seizures. 1.5-T axial T2W (a), FLAIR (b) and diffusionweighted (c) images with an ADC map (d) show subtle T2-FLAIR hyperintensity in the right amygdala and hippocampus with diffusion

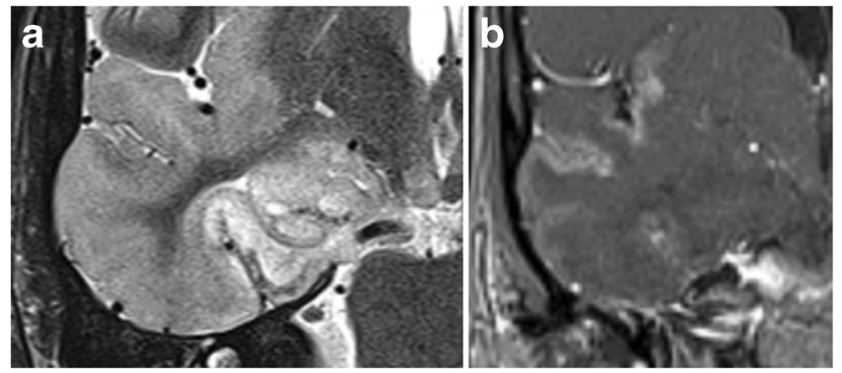

Fig. 15 Herpes encephalitis. On coronal T2-weighted images (a) extensive corticosubcortical oedema is seen in the right temporal and insular lobe. Notice the presence of cortical T2-hypointense abnormalities in the right hippocampus and along the right collateral sulcus, denoting small petechial haemorrhages. On contrast, enhanced T1-weighted images (b), there is extensive right temporal and insular gyral enhancement

distinction of $\mathrm{AD}$ from other dementia syndromes is limited however, as MTA not only occurs in AD, but can be seen in
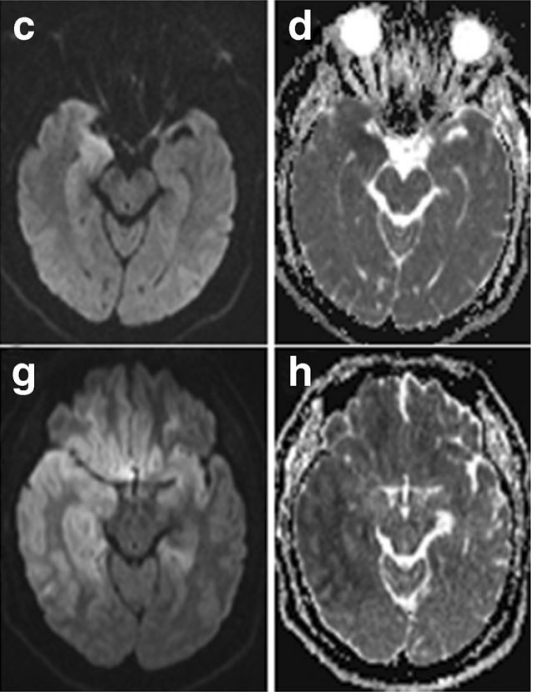

restriction. Follow-up MRI performed one week later (e-h) shows more pronounced T2-hyperintense bilateral frontotemporal cortical oedema and diffusion restriction, more pronounced in the right hemisphere. PCR was positive for HSV

other dementia syndromes as well (Fig. 12) [22]. In this regard, it is important to note that the combined visual assessment of mesial temporal atrophy and parietal atrophy has proven to not only increase the sensitivity of MRI in identifying patients with $\mathrm{AD}$, but also increase the specificity of MRI in discriminating $\mathrm{AD}$ from other dementia syndromes [23] (Fig. 13; Table 2).

\section{Infection and inflammation}

\section{Herpes simplex encephalitis}

The hippocampus can also be affected by infectious and inflammatory conditions, the best known of which is herpes simplex encephalitis (HSE). HSE is almost always caused by the HSV type 1 virus, except in neonates where type 2 predominates. The precise sequence of events leading to
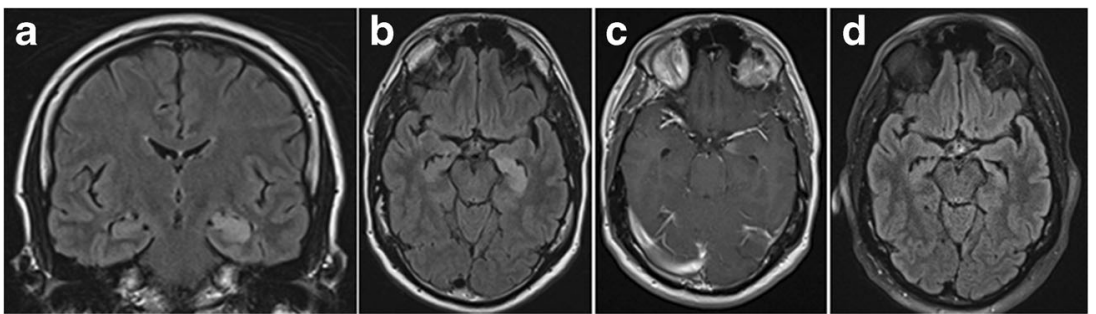

Fig. 16 Limbic encephalitis. 1.5-T coronal FLAIR (a), axial FLAIR (b) and contrast-enhanced T1W images (c) show T2-FLAIR-hyperintense swelling of the left hippocampus and discrete patchy enhancement in the left amygdala. On axial FLAIR on follow-up MRI performed
6 months later (d), the signal abnormalities have disappeared and there is volume loss of the left hippocampus with loss of the normal hippocampal digitations of the hippocampal head 


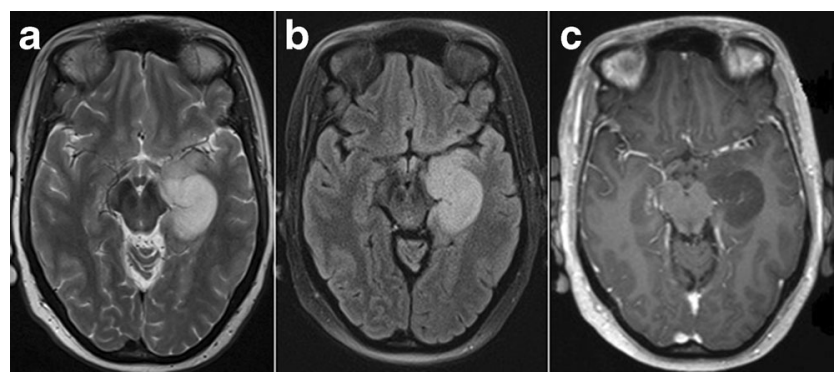

Fig. 17 Low-grade glioma. 1.5-T axial FLAIR (a), T2W (b) and contrast-enhanced $\mathrm{T} 1 \mathrm{~W}$ images (c) show a T2-hyperintense, T1hypointense, non-contrast-enhancing infiltrative mass in the left hippocampus, corresponding to a pathologically proven glioma

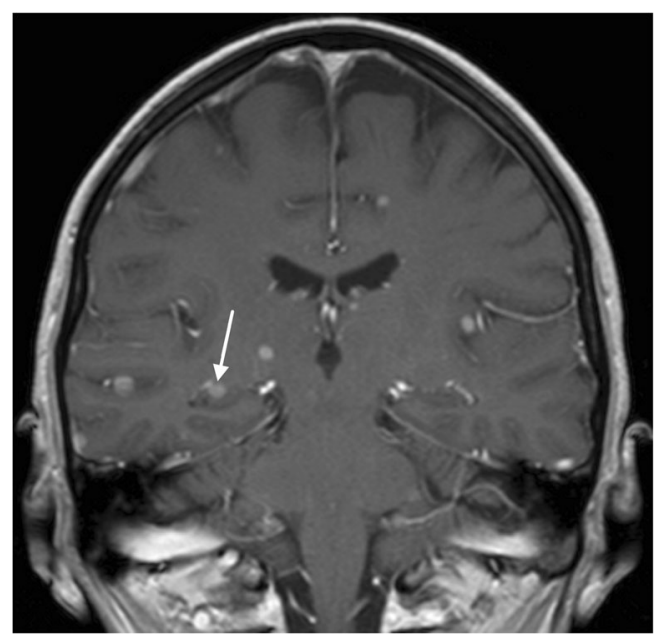

Fig. 18 Cerebral metastasis. 1.5-T coronal contrast-enhanced T1W images show multiple bilateral nodular contrast-enhancing lesions, including one in the right hippocampal body (white arrow). This was a brain metastasized bronchial carcinoma

non-neonatal HSE is unclear. It is often assumed that HSE is caused by reactivation of $\mathrm{HSV}$ in the trigeminal ganglion. An alternative hypothesis is that HSE is a primary infection rather than virus reactivation, with the olfactory system as the most probable port of entry [24]. This route of infection would better explain the typical distribution of HSE lesions in the limbic system and the insular cortex.
Symptoms reflect the propensity to involve the inferomedial frontal and temporal lobes with acute onset of hallucinations, seizures, personality changes and aphasia. HSE primarily affects neurons, which is reflected by preferential cortical involvement on MRI. On MRI, cortical abnormalities are noted as ill-defined areas of high T2/FLAIR signal intensity, usually beginning unilaterally but progressing to become bilateral. Diffusion restriction is not always present, but can be one of the earliest signs of HSE (Fig. 14) [25, 26]. Gyral enhancement and petechial haemorrhages may also be present (Fig. 15).

HSE has a bad prognosis and quick antiviral treatment is mandatory. The mortality rate of HSE exceeds $70 \%$ in patients who had no or incomplete treatment and fewer than $3 \%$ of all patients return to normal function after recovering from their illness [27].

\section{Limbic encephalitis}

Limbic encephalitis (LE) is an autoimmune-mediated paraneoplastic or non-paraneoplastic syndrome characterized by the subacute onset of confusion, anterograde amnesia, temporal lobe seizures and behavioural changes. Neuronal antibodies associated with LE can be classified into two groups according to the location of the antigen: inside the neuron or in the cell membrane [28]. The antineuronal antibodies associated with LE are anti-HU, anti-CV2, anti-Ma2 and antiamphiphysin. These antibodies are generally associated with neoplasia, specifically with small cell lung carcinoma (SCLC), breast carcinoma, testicular tumours and ovarian teratomas. The antibodies against neuronal surface antigens associated with LE include voltage-gated potassium channels (VGKC), AMPA and $\gamma$-aminobutyric acid (GABA) and are often non-paraneoplastic.

On MRI, LE manifests with uni- or bilateral swelling and varying degrees of high T2/FLAIR signal intensity in the mesial temporal lobe. Patchy enhancement and restricted diffusion can be seen. These changes may eventually evolve to medial temporal lobe atrophy and
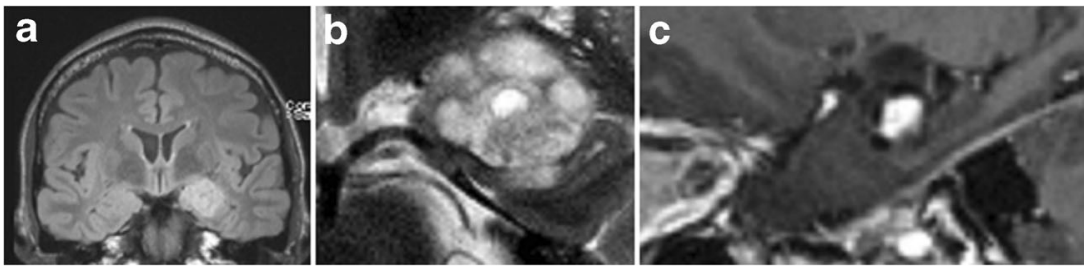

Fig. 19 Ganglioglioma. Coronal FLAIR (a), zoomed-in coronal T2 (b) and zoomed-in sagittal contrast-enhanced 3D-MPRAGE (c) show a lobulated T2-FLAIR hyperintense space-occupying lesion in the left amygdala with focal infiltration of the left hippocampal head. The lesion contains a small central cystic (b) as well as a nodular contrastenhancing component (c) 

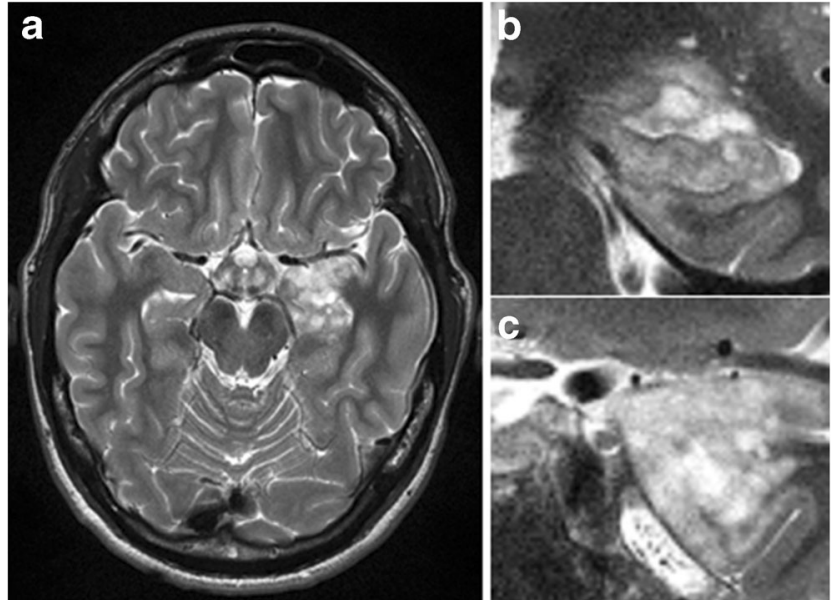

Fig. 20 DNET. Axial T2 (a) and zoomed-in coronal T2 at the level of the hippocampal head (b) and amygdala (c) show a multicystic nonenhancing (images not shown) lesion with a bubbly appearance in the cortex and subcortical white matter of the left mesiotemporal lobe infiltrating the left amygdala and hippocampal head

increased T2/FLAIR signal intensity in the hippocampus due to gliosis (Fig. 16). Imaging findings can be subtle early in the course of the disease, however, and can even remain normal in a subset of cases [29].

\section{Tumours}

Theoretically, any primary or secondary brain tumour can affect the hippocampus (Fig. 17 and 18), but a specific subgroup of tumours that are more frequently encountered in the mesial temporal lobe are the so-called longstanding epilepsyassociated tumours, such a ganglioglioma and dysembryoplastic neuroepithelial tumours (DNET; Figs. 19 and 20) [30]. Nevertheless, even these tumours rarely occur primarily in the hippocampus and are most frequently found in the parahippocampal and lateral occipitotemporal gyrus, at
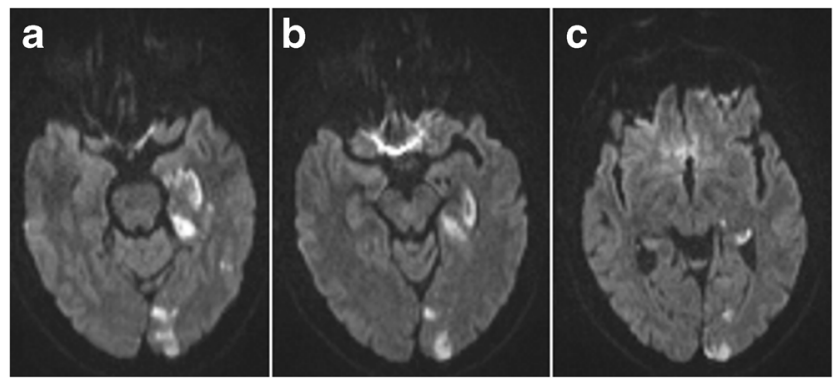

Fig. 21 Hippocampal infarction at the level of the longitudinal terminal artery segments in posterior cerebral artery stroke. Axial diffusionweighted images at the level of the hippocampal head (a), body (b) and tail (c) show diffusion restriction laterally in the hippocampus extending from the body to tail at the level of the longitudinal terminal segments, as well as several cortical diffusion-restrictive foci in the left occipital lobe
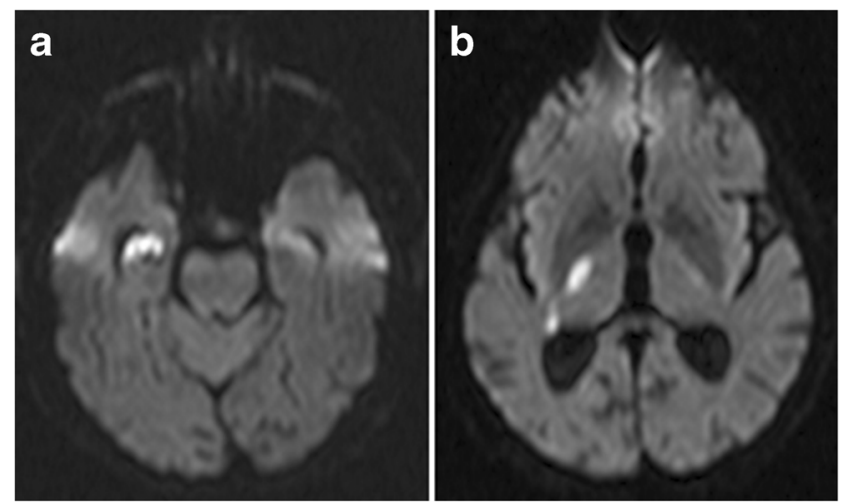

Fig. 22 Infarction of the hippocampal head in anterior choroidal artery stroke. Axial diffusion-weighted images at the level of the hippocampal head (a) and the basal ganglia (b) show diffusion restriction in the hippocampal head and in the posterior limb of the internal capsule

the transition areas from allocortex to neocortex [31]. Ganglioglioma and DNET are found mostly in children and young adults.

Gangliogliomas are located in the cortex or in the cortex and subcortical white matter. The classical presentation of ganglioglioma on MRI, seen in about $40 \%$ of cases, consists of a well-defined cystic mass with a mural nodule. A solid mass showing low to intermediate signal intensity on T1W and high signal intensity on $\mathrm{T} 2 \mathrm{~W}$ images is also not uncommon. Calcifications are present in about $30 \%$ of cases. Enhancement of the solid portion of the tumour is variable, ranging from non-enhancement (50\% of cases) to ringlike enhancement to homogeneous enhancement. Perilesional ede$\mathrm{ma}$ is absent or limited [30].

On MRI, DNET usually appear as multilobulated or gyriform cysts which are located in the cortex or in the cortex and subcortical white matter. The multilobulated cysts are characteristically hypointense on T1- and strongly hyperintense on T2W images. On FLAIR images, they have mixed signal intensity and often a peripheral rim of high signal intensity, the so-called "bright rim sign", can be seen. Enhancement is seen in about $30 \%$ of cases, calcifications in less than $20 \%$. Rarely, only a solitary large cyst is seen. Diffusion restriction is absent [30].

\section{Vascular disease}

\section{Arterial ischemic stroke}

As discussed earlier, the hippocampus has a complex arterial supply with three hippocampal arteries or groups of arteries supplying the hippocampal head, body and tail. The anterior choroidal artery has a variable contribution to the vascularization of the hippocampal head, which 
Fig. 23 64-year-old man with transient global amnesia. Axial T2W (a) and diffusion-weighted images (b) with an ADC map (c) show a small T2-hyperintense, diffusion-restrictive focus in the left hippocampal body (white arrows)
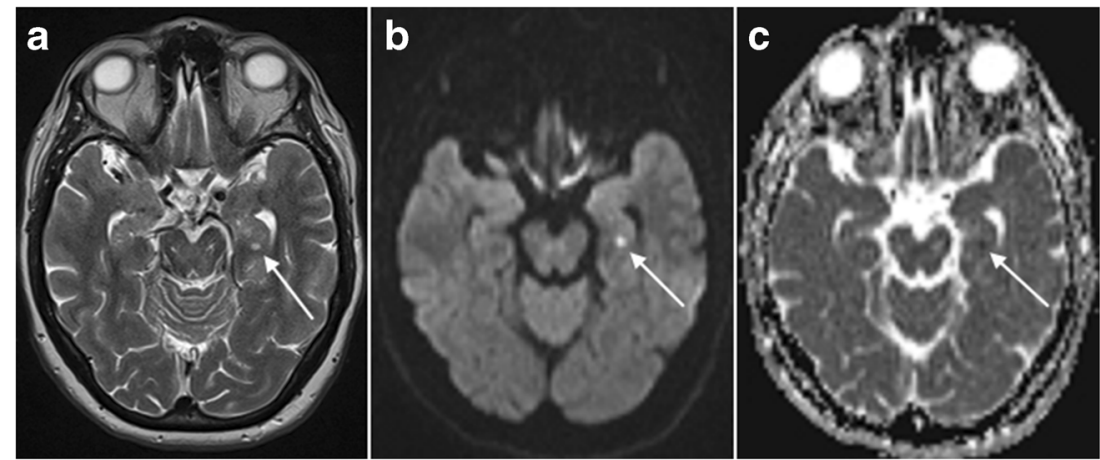

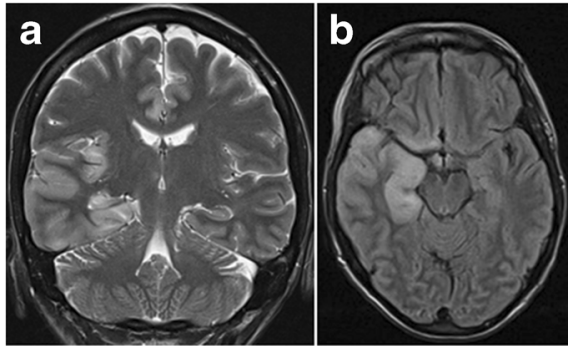

Fig. 24 Status epilepticus. A 30-year-old woman with epilepsy quit her anti-epileptic treatment during pregnancy and was brought to the hospital in status epilepticus. Coronal T2W (a) and axial FLAIR images (b) in the acute phase show extensive cortical oedema in the right temporal lobe with involvement of the right hippocampus as well as in the right insula. There was no diffusion restriction or contrast enhancement (images not shown). Control MRI performed 4 days later after initiation of anti-

may be preponderant in some cases [1]. Hence, the hippocampal head can be involved in anterior choroidal artery infarctions and the entire hippocampus in posterior cerebral artery infarctions (Figs. 21 and 22) [32]. Of note is that infarction limited to the hippocampus is rare and in acute hippocampal infarction, additional extrahippocampal infarc-
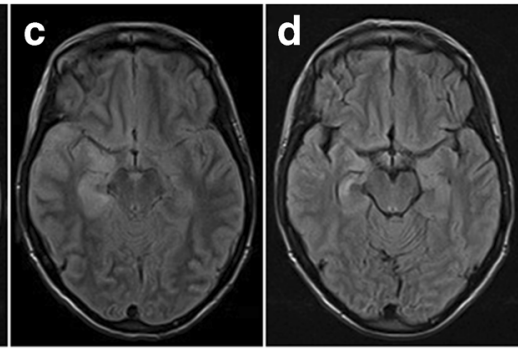

epileptic treatment shows regression of the oedema on axial FLAIR (c). On follow-up MRI performed one month later, axial FLAIR shows volume loss of the right hippocampus as well as increased signal intensity, reflecting secondary gliotic changes (d). Alternatively, this could also be a preexistent mesial temporal sclerosis. As extensive clinical work-up revealed no other possible cause for the observed cortical oedema, final diagnosis was seizure-induced cortical edema
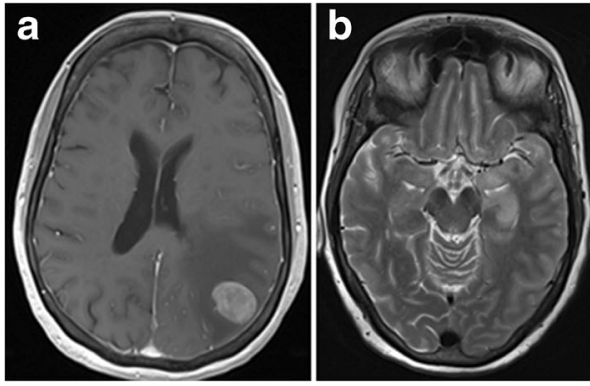

Fig. 25 Postictal edema. A 60-year-old woman with stage 4 bronchial carcinoma was admitted to the ER because of generalized epileptic seizures. Contrast-enhanced axial T1 (a) showed a contrast-enhancing mass lesion with extensive perilesional oedema in the left parietal lobe, tions in the territory of the anterior choroidal artery or posterior cerebral artery are highly likely [31]. This can be used to differentiate acute ischemic hippocampal infarction from other conditions manifesting with hippocampal diffusion restriction, such as transient global amnesia and seizure-induced abnormalities [32].

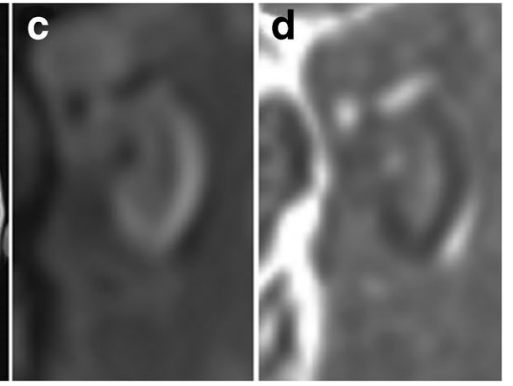

corresponding to a brain metastasis. An axial T2 image (b) shows increased signal intensity in the left hippocampus and amygdala. Diffusion-weighted images (c) with an ADC map (d) show restricted diffusion laterally in the left hippocampus 

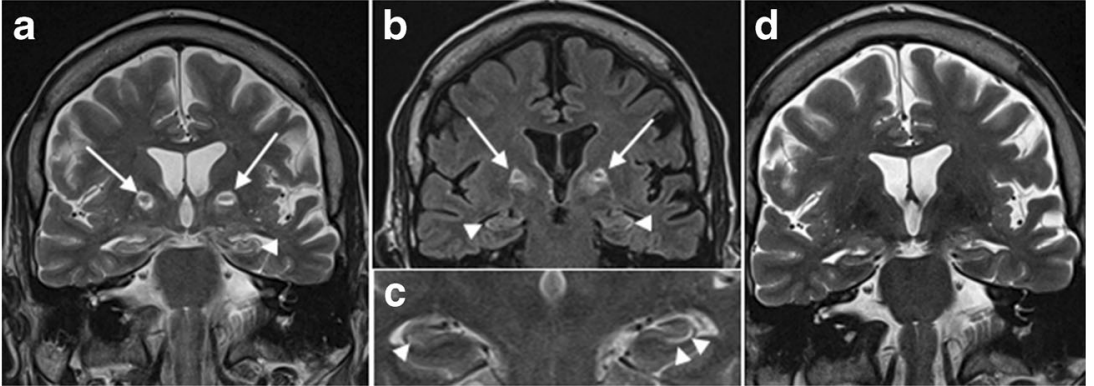

Fig. 26 Presumed carbon monoxide poisoning. A 49-year-old man was found comatose and with an alcohol intoxication in his garage next to his car, which was not running. The patient received an MRI 14 days after admittance to the hospital. Coronal T2-weighted (a,c) and FLAIR images (c) showed T2-hyperintense lesions with central iso- to hypo-intense areas in both pallidi (white arrows) as well as T2 hyper-intensity of both hippocampi from head to tail involving the CA2 sector on the right and the CA1 and CA2 sectors on the left (white arrowheads). On

\section{Transient global amnesia}

Transient global amnesia is a clinical syndrome characterized by acute retrograde amnesia, generally occurring in patients older than 60 years of age and lasting less than 24 hours. Its aetiology is unclear and several hypotheses have been formulated, including arterial thrombo-embolism, venous congestion, epilepsy and migraine. Although the diagnosis is mainly clinical, the detection of uni- or bilateral small punctate foci of diffusion restriction on MR imaging can help to confirm it. On MRI, hippocampal signal abnormalities can be found in up to $85 \%$ of patients, depending on the used MRI parameters and the time elapsed from onset (Fig. 23) [33]. follow-up MRI performed 4 months later, these abnormalities had disappeared (d). The patient suffered from amnesia and the details of what happened exactly remain a mystery to this date. The patient was suicidal and the abnormalities in the pallidi are very suggestive for carbon monoxide poisoning, however, with the hippocampal abnormalities reflecting damage to the especially vulnerable Sommers CA2 sector of the hippocampi

\section{Toxic-metabolic diseases}

Due to the vulnerability of the hippocampus to excitotoxic brain injury and its primary or secondary involvement in many types of epileptic seizures, the hippocampus is the most frequent location of acute seizure-induced brain abnormalities, which, on MRI, manifest as increased cortical signal intensity on T2W and FLAIR images with or without diffusion restriction (Figs. 24 and 25) [34]. When diffusion restriction is present, it does not automatically imply neuronal cell death, as seizure-induced diffusion-restrictive lesions often show reversibility and are, therefore, sustained by conditions different from ischemic cytotoxic edema [34]. The susceptibility of the

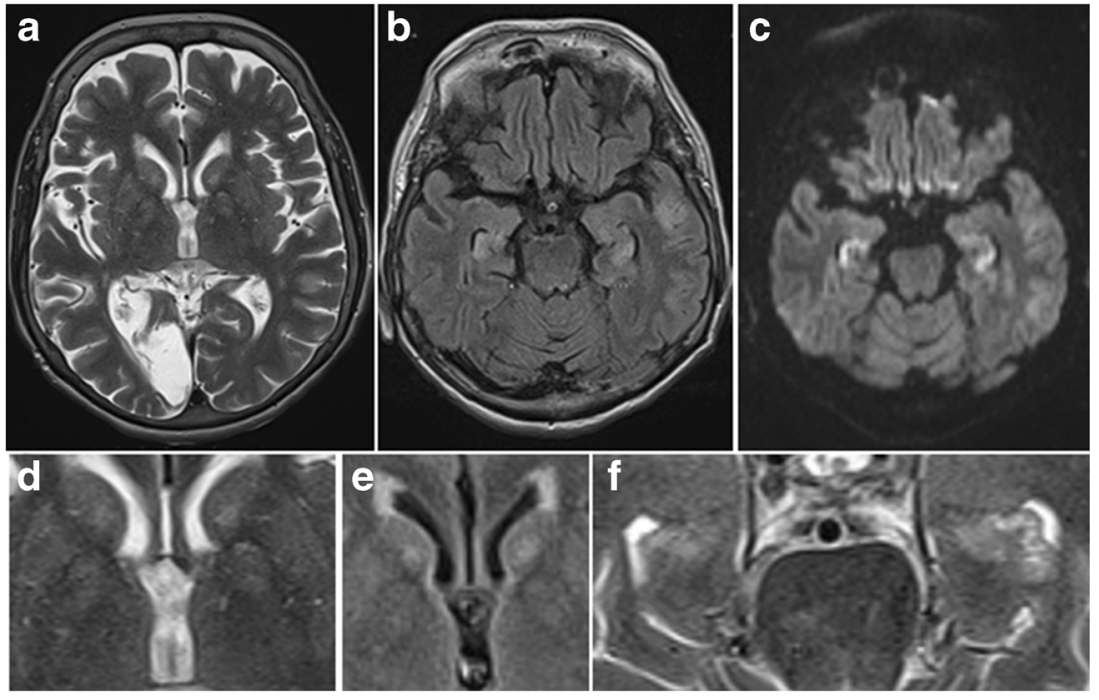

Fig. 27 Hypoglycemic encephalopathy. A 60-year-old diabetic woman with an old right occipital infarction tried to commit suicide by overdosing on insulin. MRI performed 7 days after being admitted to the intensive care unit shows subtle patchy increased T2 signal in the bilateral caudate and lentiform nuclei (a), which is better appreciated on the zoomed-in axial T2 and FLAIR images (d, e). Increased T2-FLAIR signal intensity and restricted diffusion is also observed in both hippocampi (b, c). Zoomed-in axial T2 images of the hippocampal head nicely illustrate the increased $\mathrm{T} 2$ signal (f) 
hippocampus to excitotoxic or hypoxic brain injury also explains its involvement in many kinds of toxic-metabolic disorders, a detailed description of which is beyond the scope of this review and, as these abnormalities are generally bilateral, have already been described more extensively in pictorial reviews focusing on bilateral temporal lobe disease (Fig. 26 and 27) [35].

\section{Conclusion}

MRI is the preferred imaging technique to assess hippocampal anatomy and pathology. The main indications for tailored depiction of the hippocampus are mesial temporal sclerosis and dementia. A wide range of pathologic conditions with often similar imaging characteristics can be centred in and around the hippocampus, however, and correlation with clinical data is often necessary to come to a correct diagnosis or formulate a sound differential diagnosis.

\section{Compliance with ethical standards}

Statements Martin Wiesmann has the following disclosures:

- Grants: Stryker Neurovascular. Siemens Healthcare

- Personal fees: Stryker Neurovascular. Silkroad Medical. Siemens Healthcare. Bracco

- Non-financial support: Codman Neurovascular. Covidien. Abbott. St. Jude Medical. Phenox. Penumbra. Microvention/Terumo. B. Braun. Bayer. Acandis. ab medica

The other authors have nothing to disclose.

The article received no grants or funding.

Open Access This article is distributed under the terms of the Creative Commons Attribution 4.0 International License (http:// creativecommons.org/licenses/by/4.0/), which permits unrestricted use, distribution, and reproduction in any medium, provided you give appropriate credit to the original author(s) and the source, provide a link to the Creative Commons license, and indicate if changes were made.

\section{References}

1. Duvernoy HM (2005) The Human Hippocampus: Functional Anatomy, Vascularization and Serial Sections with MRI, 3rd edn. Springer Verlag, Berlin

2. von Bechterew W (1900) Demonstration eines Gehirns mit Zerstörung der vorderen und inneren Theile der Himrinde beider Schlafenlappen. Neurologisch Zentralbl 19:990-999

3. Scoville WB, Milner B (1957) Loss of recent memory after bilateral hippocampal lesions. J Neurol Neurosurg Psychiatry 120:11-21

4. Deblaere K, Achten E (2008) Structural magnetic resonance imaging in epilepsy. Eur Radiol 18:119-129

5. Barkhof F, Fox NC, Bastos-Leite AJ, Scheltens P (2011) Neuroimaging in dementia. Springer-Verlag, Berlin Heidelberg

6. Phal PM, Usmanov A, Nesbit GM, Anderson JC, Spencer D, Wang P, Helwig JA, Roberts C, Hamilton BE (2008)
Qualitative comparison of 3-T and 1.5-T MRI in the evaluation of epilepsy. AJR Am J Roentgenol 191:890-895

7. Naganawa S, Koshikawa T, Nakamura T, Kawai H, Fukatsu H, Ishigaki T, Komada T, Maruyama K, Takizawa O (2004) Comparison of flow artifacts between 2D-FLAIR and 3D-FLAIR sequences at 3 T. Eur Radiol 14:1901-1908

8. Achten E, Boon P, De Poorter J, Calliauw L, Van de Kerckhove T, De Reuck J, Kunnen M (1995) An MR protocol for presurgical evaluation of patients with complex partial seizures of temporal lobe origin. AJNR Am J Neuroradiol 16:1201-1213

9. Tubbs RS, Muhleman M, McClugage SG, Loukas M, Miller JH, Chern JJ, Rozzelle CJ, Oakes WJ, Cohen-Gadol AA (2012) Progressive symptomatic increase in the size of choroidal fissure cysts. J Neurosurg Pediatr 10:306-309

10. Bajic D, Kumlien E, Mattsson P, Lundberg S, Wang C (2009) Raininko R (2009) Incomplete hippocampal inversion-is there a relation to epilepsy? Eur Radiol 19:2544-2550

11. Bajic D, Wang C, Kumlien E, Mattsson P, Lundberg S, Eeg-Olofsson O, Raininko R (2008) Incomplete inversion of the hippocampus-a common developmental anomaly. Eur Radiol 18:138-142

12. Chew AP, Gupta G, Alatakis S, Schneider-Kolsky M, Stuckey SL (2012) Hippocampal calcification prevalence at CT: a retrospective review. Radiology 265:504-510

13. Blümcke I, Thom M, Wiestler OD (2002) Ammon's horn sclerosis: a maldevelopmental disorder associated with temporal lobe epilepsy. Brain Pathol 12:199-211

14. Cendes F (2004) Febrile seizures and mesial temporal sclerosis. Curr Opin Neurol 17:161-164

15. Bote RP, Blázquez-Llorca L, Fernández-Gil MA, AlonsoNanclares L, Muñoz A, De Felipe J (2008) Hippocampal sclerosis: histopathology substrate and magnetic resonance imaging. Semin Ultrasound CT MR 29:2-14

16. Chan S, Erickson JK, Yoon SS (1997) Limbic system abnormalities associated with mesial temporal sclerosis: a model of chronic cerebral changes due to seizures. Radiographics 17:1095-1110

17. Oikawa H, Sasaki M, Tamakawa Y, Kamei A (2001) The circuit of Papez in mesial temporal sclerosis: MRI. Neuroradiology 43: 205-210

18. Camacho DL, Castillo M (2007) MR imaging of temporal lobe epilepsy. Semin Ultrasound CT MR 28:424-436

19. Salanova V, Markand O, Worth R (2004) Temporal lobe epilepsy: analysis of patients with dual pathology. Acta Neurol Scand 109: 126-131

20. Visser PJ, Verhey FR, Hofman PA, Scheltens P, Jolles J (2002) Medial temporal lobe atrophy predicts Alzheimer's disease in patients with minor cognitive impairment. J Neurol Neurosurg Psychiatry 72:491-497

21. Scheltens P, Leys D, Barkhof F, Huglo D, Weinstein HC, Vermersch P, Kuiper M, Steiling M, Wolters EC, Valk J (1992) Atrophy of medial temporal lobes on MRI in "probable" Alzheimer's disease and normal ageing: diagnostic value and neuropsychological correlates. J Neurol Neurosurg Psychiatry 55:967-972

22. Wahlund LO, Julin P, Johansson SE et al (2000) Visual rating and volumetry of the medial temporal lobe on magnetic resonance imaging in dementia: a comparative study. J Neurol Neurosurg Psychiatry 69:630-635

23. Koedam ELGE, Lehmann M, van der Flier WM, Scheltens P, Pijnenburg YAL, Fox N, Barkhof F, Wattjes MP (2011) Visual assessment of posterior atrophy development of a MRI rating scale. Eur Radiol 21:2618-2625

24. Baskin HJ, Hedlund G (2007) Neuroimaging of herpesvirus infections in children. Pediatr Radiol 37:949-963

25. Heiner L, Demaerel P (2003) Diffusion-weighted MR imaging findings in a patient with herpes simplex encephalitis. Eur J Radiol 45:195-198 
26. Küker W, Nägele T, Schmidt F, Heckl S, Herrlinger U (2004) Diffusion-weighted MRI in herpes simplex encephalitis: a report of three cases. Neuroradiology 46:122-125

27. Steiner I, Budka H, Chaudhuri A et al (2010) Viral meningoencephalitis: a review of diagnostic methods and guidelines for management. Eur J Neurol 17:999. e57

28. Graus F, Saiz A, Dalmau J (2010) Antibodies and neuronal autoimmune disorders of the CNS. J Neurol 257:509-517

29. Demaerel P, Van Dessel W, Van Paesschen W, Vandenberghe R, Van Laere K, Linn J (2011) Autoimmune-mediated encephalitis. Neuroradiology 53:837-851

30. Urbach H (2008) MRI of long-term epilepsy-associated tumors. Semin Ultrasound CT MR 29:40-46
31. Urbach H (2006) Der Hippokampus. Radiologie up2date 6:359368

32. Szabo K, Förster A, Jäger T, Kern R, Griebe M, Hennerici MG, Gass A (2009) Hippocampal lesion patterns in acute posterior cerebral artery stroke: clinical and MRI findings. Stroke 40:20422045

33. Bartsch T, Deuschl G (2010) Transient global amnesia: functional anatomy and clinical implications. Lancet Neurol 9:205-214

34. Cianfoni A, Caulo M, Cerase A, Della Marca G, Falcone C, Di Lella GM, Gaudino S, Edwards J, Colosimo C (2013) Seizureinduced brain lesions: a wide spectrum of variably reversible MRI abnormalities. Eur J Radiol 82:1964-1972

35. Eran A, Hodes A, Izbudak I (2016) Bilateral temporal lobe disease: looking beyond herpes encephalitis. Insights Imaging 7(2):265-274 